\title{
SUBNUTRIÇÃO EMIDOSOS COM DEMÊNCIA: ATENÇÃO AOS ESTÁGIOS AVANÇADOS DA DOENÇA
}

Malnutrition in the elderly with dementia: attention to advanced stages

\author{
Priscila Cansian Rossin ${ }^{a}$, Patricia Viganó Contri Degiovannia, Júlio Cesar Morigutia
}

OBJETIVO: Caracterizar o estado nutricional de idosos com demência em diferentes estágios da doença e identificar fatores associados com a subnutrição nesse grupo. MÉTODOS: Estudo transversal realizado com 92 idosos demenciados em diferentes estágios da doença, de acordo com o Clinical Dementia Rating (CDR: 1-3). Foram avaliados dados demográficos, clínicos, antropométricos e exames laboratoriais relacionados ao estado nutricional, sendo que o diagnóstico nutricional foi determinado por uma combinação de fatores antropométricos e exames laboratoriais. RESULTADOS: Do total, 64,1\% eram mulheres e a média de idade do grupo foi de 80,2 \pm 6,8 anos. De acordo com o estado nutricional, 52,1\% dos pacientes foram classificados como subnutridos, sendo encontradas diferenças significativas entre as medidas antropométricas e os grupos. Foi observado que idosos com demência moderada e avançada apresentaram maior risco de subnutrição do que idosos com demência em estágio inicial $(p=0,04)$, sendo que os idosos com história de perda ponderal involuntária apresentaram chance três vezes maior de desenvolver subnutrição. CONCLUSÃO: A subnutrição foi identificada em mais da metade dos idosos com demência estudados, sendo que o comprometimento cognitivo avançado e a perda ponderal recente estão diretamente associados com o prejuízo do estado nutricional desse grupo de idosos.

PALAVRAS-CHAVE: idoso; demência; estado nutricional; desnutrição.

OBJECTIVE: To characterize the nutritional status of elderly people with dementia at different stages of the disease and to identify factors associated with malnutrition in this group. METHODS: Cross-sectional observational study with 92 elderly demented in various stages of the disease according to the Clinical Dementia Rating (CDR: 1-3). Demographic, clinical and anthropometric data, as well as laboratory tests related to nutritional status were evaluated; the nutritional diagnosis was determined using a combination of laboratory and anthropometric measurements. RESULTS: Of the total, $64.1 \%$ were female and the mean age of the group was $80.2 \pm 6.8$ years. According to nutritional status, $52.1 \%$ of the patients were classified as undernourished, being found significant differences between the anthropometric measures and the groups. It was observed that elderly with moderate and advanced dementia had higher risk of malnutrition than elderly with dementia an initial stage $(p=0.04)$; the elderly with history of unintentional weight loss presented three times greater chance of developing malnutrition. CONCLUSION: The malnutrition was identified in more than half of elderly people with dementia; the advanced cognitive impairment and recent weight loss are directly associated with the injury of the nutritional status of this group of elderly.

KEYWORDS: aged; dementia; nutritional status; malnutrition. 


\section{INTRODUÇÃO}

A demência é uma das mais devastadoras doenças associadas com dano cognitivo; acarreta mudanças significativas para o paciente, família e sociedade. $O$ envelhecimento da população mundial tem elevado a prevalência de danos e transtornos cognitivos. ${ }^{1}$ Espera-se que problemas relacionados às demências aumentem principalmente em países de média e baixa renda, devendo atingir 115,4 milhões de indivíduos no mundo em $2050 .^{2}$

O estado nutricional é comprometido entre os idosos como um todo, sendo observada elevada prevalência de subnutrição. ${ }^{3}$ Dessa forma, os idosos com demência apresentam, além das alterações associadas ao processo de envelhecimento, limitações cognitivas, físicas e alterações de comportamento que podem comprometer ainda mais o estado nutricional desses indivíduos. ${ }^{4}$ Quando comparado com indivíduos sem alteração cognitiva, os idosos com demência apresentam valores menores de peso, índice de massa corporal (IMC), massa magra e reserva adiposa. ${ }^{5}$

A perda de peso involuntária é um fator de risco nutricional que parece preceder o início dos sintomas de demência por vários anos e que se agrava à medida que a doença progride. ${ }^{6}$ Entretanto, os mecanismos envolvidos na perda ponderal observada nos idosos com alteração cognitiva ainda não foram completamente elucidados.

Nesse contexto, é importante identificar precocemente o risco nutricional entre esses indivíduos, uma vez que a perda ponderal e a subnutrição ocasionam uma série de complicações que levam ao aumento da incidência de úlceras por pressão, infecção, falência respiratória, insuficiência cardíaca e tempo de internação hospitalar, os quais podem agravar o prognóstico da doença, resultando em maiores taxas de morbidade e mortalidade. ${ }^{7}$

Dessa forma, o objetivo deste estudo foi caracterizar o estado nutricional de idosos com demência em diferentes estágios da doença e identificar fatores associados com a subnutrição nesse grupo de pacientes.

\section{MÉTODOS}

O presente trabalho, de caráter descritivo e transversal, foi constituído por 92 idosos de ambos os gêneros, acompanhados nos ambulatórios de geriatria de um hospital universitário.

O único critério de inclusão utilizado foi a presença do diagnóstico de demência segundo os critérios do Diagnostic and Statistical Manual of Mental Disorders (DSM IV), ${ }^{8}$ nos seus diferentes estágios de progressão, conforme classificação do Clinical Dementia Rating (CDR). ${ }^{9}$ Os idosos portadores de hemiplegia, edema, ascite, câncer que não foi considerado curado ou em remissão, e aqueles com insuficiência cardíaca grave foram excluídos do estudo, uma vez que essas condições podem interferir diretamente no diagnóstico do estado nutricional. Dessa forma, foram incluídos 30 idosos com demência leve (CDR 1), 30 com demência moderada (CDR 2) e 32 com demência avançada (CDR 3).

O estudo foi aprovado pelo Comitê de Ética em pesquisa (Processo no 2960/2009), sendo que os responsáveis pelos idosos com demência que aceitaram participar do estudo assinaram o Termo de Consentimento Livre e Esclarecido.

Todos os indivíduos foram submetidos à avaliação antropométrica, sendo obtidas as medidas de peso corporal, altura, circunferência braquial $(\mathrm{CB})$, circunferência da panturrilha $(\mathrm{CP})$ e prega cutânea tricipital (PCT) de acordo com procedimentos padrão. ${ }^{10}$ Foram calculados os valores do $\mathrm{IMC}\left(\mathrm{kg} / \mathrm{m}^{2}\right)$ e da circunferência muscular do braço $(\mathrm{CMB}, \mathrm{em} \mathrm{cm})$.

Para os idosos impossibilitados, o peso e altura foram estimados pelas equações de Chumlea et al. ${ }^{11,12}$ Utilizou-se como referência para análise do percentual de adequação das medidas de CB, CMB, CP e PCT os dados do estudo Saúde, Bem-estar e Envelhecimento (SABE). ${ }^{13}$

Os exames laboratoriais - albumina sérica, hemoglobina, hematócrito e colesterol - foram realizados conforme os métodos padronizados de análise do local do estudo e obtidos dos prontuários do paciente.

A classificação do estado nutricional foi realizada por meio da combinação de parâmetros antropométricos e laboratoriais, o que aumenta a sensibilidade da avaliação nutricional dos idosos. ${ }^{14,15}$ Os indivíduos foram classificados como subnutridos quando os valores de três ou mais parâmetros nutricionais avaliados estavam abaixo da normalidade, incluindo pelo menos um parâmetro antropométrico e um laboratorial. Foram considerados valores abaixo da normalidade a porcentagem de adequação menor do que $90 \%$ para peso corporal, PCT e $\mathrm{CMB}$, valores inferiores a $23 \mathrm{~kg} / \mathrm{m}^{2}$ para IMC, $3,5 \mathrm{~g} / \mathrm{dL}$ para albumina e $150 \mathrm{mg} / \mathrm{dL}$ para colesterol, para ambos os sexos; valores de hemoglobina de 14 e $12 \mathrm{~g} / \mathrm{dL}$ e hematócrito de 40 e $35 \%$ para homens e mulheres, respectivamente. Para os idosos classificados como não subnutridos, foi utilizado o IMC para o diagnóstico de eutrofia ou excesso de peso corporal, de acordo com os pontos de corte recomendados pela Organização PanAmericana de Saúde (OPAS). ${ }^{16}$

Fatores sociodemográficos (idade, gênero, local de residência, responsável pelos cuidados), clínicos (etiologia da demência, estágio de progressão da doença, polifarmácia - mais do que 3 medicamentos/dia - presença de perda ponderal recente, úlcera por pressão) e dados relacionados à alimentação (dificuldade de mastigação e deglutição, necessidade de modificação na consistência da dieta e dependência para alimentação) foram 
coletados para a avaliação dos fatores associados à subnutrição dos idosos com demência envolvidos no estudo.

A avaliação estatística foi realizada utilizando o software SAS 9.0. As variáveis quantitativas foram descritas como média \pm desvio padrão (DP), enquanto as variáveis qualitativas, como frequência e porcentagem. A classificação do estado nutricional foi utilizada para definição dos grupos: subnutridos e não subnutridos.

Dessa forma, as diferenças quantitativas entre os grupos foram determinadas pelo teste $t$ de Student, enquanto para comparação das variáveis categóricas utilizou-se o teste exato de Fisher ou teste do $\chi^{2}$. O nível de significância foi estabelecido em $5 \%$ em todas as análises.

A fim de verificar a associação entre a presença de subnutrição e os fatores sociodemográficos, clínicos e relacionados à alimentação foi aplicada a metodologia de regressão logística, obtendo-se medidas de razão de chances (RC) e seus intervalos de confiança 95\% (IC95\%). O modelo de regressão foi ajustado pelas variáveis: idade, gênero e gravidade da demência (CDR).

\section{RESULTADOS}

Entre os 92 idosos avaliados, 64,1\% eram mulheres, não existindo diferenças em relação à distribuição de gênero entre os grupos conforme a classificação do estado nutricional.
A idade variou de 67 a 96 anos, sendo que a média de idade de todos os idosos foi de $80,2 \pm 6,8$ anos. Não foram observadas diferenças na média de idade entre os grupos (subnutridos: 80,2 $\pm 6,3$; não subnutridos: 79,8 $\pm 6,5 ; \mathrm{p}=0,59)$.

A demência devida à doença de Alzheimer foi a mais prevalente entre os idosos $(n=63 ; 68,4 \%$ do grupo), seguida pela demência mista $(n=19 ; 20,7 \%)$, demência vascular $(n=6 ; 6,5 \%)$ e pela demência com corpos de Lewy $(n=4 ; 4,4 \%)$.

Não houve associação entre a presença de subnutrição e as variáveis idade acima de 80 anos, gênero, local de residência e responsável pelos cuidados (Tabela 1). Em todos os grupos foi observado que os idosos residiam, principalmente, em domicílio com um familiar, sendo o filho o principal responsável pelo cuidado dos mesmos. Nenhum dos idosos com demência avançada residia sozinho.

Não houve associação entre a etiologia da demência e o estado nutricional dos idosos com demência. No entanto, a gravidade do comprometimento cognitivo está diretamente associada com o prejuízo do estado nutricional, em que idosos com demência moderada e avançada apresentam maior risco de subnutrição do que idosos com demência em estágio inicial. Da mesma forma, idosos com história de perda ponderal recente apresentam chance três vezes maior de desenvolver subnutrição (Tabela 2).

No presente estudo, não houve associação entre o uso de múltiplos medicamentos, a necessidade de modificação da

Tabela 1 Prevalência e razão de chances entre as variáveis sociodemográficas e os grupos segundo diagnóstico de subnutrição, São Paulo, 2015 ( $n$ = 92)

\begin{tabular}{|c|c|c|c|c|c|}
\hline Variável & $\begin{array}{l}\text { Subnutrido } \\
(\mathrm{n}=48) \\
\mathrm{n}(\%)\end{array}$ & $\begin{array}{l}\text { Não subnutrido } \\
\qquad \begin{array}{c}(\mathrm{n}=44) \\
\mathrm{n}(\%)\end{array}\end{array}$ & Valor $p$ & $\begin{array}{l}\text { Razão de } \\
\text { chance }\end{array}$ & IC95\% \\
\hline Idade ${ }^{a}$ & & & 0,45 & & \\
\hline$\geq 80$ anos & $26(54,1)$ & $21(47,7)$ & & 1,17 & $0,49-2,76$ \\
\hline$<80$ anos & $22(45,8)$ & $23(52,2)$ & & - & - \\
\hline Gênero ${ }^{a}$ & & & 0,53 & & \\
\hline Masculino & $17(35,4)$ & $15(34,1)$ & & 1,03 & $0,41-2,55$ \\
\hline Feminino & $31(64,5)$ & $29(65,9)$ & & & \\
\hline Residência ${ }^{a}$ & & & 0,54 & & \\
\hline Familiar & $38(79,1)$ & $39(88,6)$ & & - & - \\
\hline Sozinho & $1(2,0)$ & $2(4,6)$ & & 1,1 & $0,21-5,74$ \\
\hline ILP & $6(12,5)$ & $3(6,8)$ & & 0,93 & $0,16-5,36$ \\
\hline Responsável pelo cuidado & & & 0,47 & & \\
\hline Filho & $24(50,0)$ & $26(59,1)$ & & - & - \\
\hline Cônjuge & $14(29,1)$ & $14(31,8)$ & & 1,76 & $0,6-5,11$ \\
\hline Cuidador formal & $10(20,8)$ & $4(9,1)$ & & 2,32 & $0,64-847$ \\
\hline
\end{tabular}

IC95\%: intervalo de confiança de 95\%; ${ }^{a}$ teste do $\chi^{2}$; ILP: instituição de longa permanência. 
Tabela 2 Prevalência e razão de chances entre as variáveis clínicas e relacionadas à alimentação e os grupos segundo diagnóstico de subnutrição, São Paulo, 2015 ( $n$ = 92)

\begin{tabular}{|c|c|c|c|c|c|}
\hline Variável & $\begin{array}{l}\text { Subnutrido } \\
\text { (n = 48) } \\
n(\%)\end{array}$ & $\begin{array}{l}\text { Não subnutrido } \\
\qquad \begin{array}{c}(n=44) \\
n(\%)\end{array}\end{array}$ & Valor $\mathbf{p}$ & $\begin{array}{l}\text { Razão de } \\
\text { chance }\end{array}$ & IC95\% \\
\hline Etiologia da demência - DDA & $32(71,1)$ & $29(65,9)$ & 0,97 & 0,81 & $0,13-4,79$ \\
\hline \multicolumn{6}{|l|}{ Gravidade da demência ${ }^{a}$} \\
\hline CDR 1 & $11(36,7)$ & $19(63,3)$ & \multirow{3}{*}{0,04} & - & - \\
\hline CDR 2 & $15(50,0)$ & $15(50,0)$ & & 1,73 & $0,62-4,88$ \\
\hline CDR 3 & $22(68,8)$ & $10(31,2)$ & & 3,72 & $1,29-10,75$ \\
\hline Polifarmácia ${ }^{a}$ & $33(73,3)$ & $37(84,1)$ & 0,21 & 1,79 & $0,61-5,26$ \\
\hline Presença de úlcera por pressãob & $6(13,3)$ & - & 0,02 & - & - \\
\hline Perda ponderal recente ${ }^{a}$ & $38(79,1)$ & $24(54,5)$ & 0,01 & 3,03 & $1,08-8,48$ \\
\hline Dieta modificada - consistência ${ }^{a}$ & $29(60,4)$ & $17(38,6)$ & 0,39 & 1,55 & $0,56-4,32$ \\
\hline Dificuldade de mastigação ${ }^{a}$ & $29(60,4)$ & $17(38,6)$ & 0,67 & 1,34 & $0,49-3,69$ \\
\hline Dificuldade de deglutição ${ }^{a}$ & $23(47,9)$ & $12(27,2)$ & 0,05 & 1,57 & $0,57-4,32$ \\
\hline Dependência para alimentaçãoa & $19(39,5)$ & $7(15,9)$ & 0,02 & 2,34 & $0,58-9,48$ \\
\hline
\end{tabular}

IC95\%: intervalo de confiança de 95\%; DDA: demência devida à doença de Alzheimer; ateste do $\chi^{2}$; bteste exato de Fisher; CDR 1: idosos com demência leve; CDR 2: idosos com demência moderada; CDR 3: idosos com demência avançada.

consistência da dieta, a dificuldade de mastigação ou deglutição e o estado nutricional dos idosos com demência. Com relação à dependência para alimentação, quase todos os idosos com demência leve e moderada eram autônomos para realizar sua refeição, situação diferente dos idosos com demência em estágio avançado da doença, em que $23(71,8 \%)$ necessitavam de ajuda para se alimentar.

Dessa forma, foi encontrada associação significativa em relação à dependência para alimentação e os grupos, embora pela análise de regressão logística, ajustada para idade, gênero e gravidade da demência, esse achado não tenha sido confirmado.

\section{DISCUSSÃO}

De acordo com o presente estudo, a subnutrição foi observada em $52 \%$ dos idosos com demência, sendo que a perda ponderal recente e a progressão do comprometimento cognitivo estão diretamente associadas com a subnutrição.

Não existe um método único capaz de fornecer um diagnóstico preciso do estado nutricional, em especial entre os idosos, questão amplamente discutida na literatura. Alguns autores propõem que para a determinação mais acurada do estado nutricional dos idosos, é necessário avaliar a história alimentar, os sinais clínicos de subnutrição, as medidas antropométricas e sua variação temporal, além de determinações hematológicas, séricas e urinárias. ${ }^{17}$
Dentre os fatores possivelmente associados com a subnutrição, a presença de perda ponderal recente aumenta em 3 vezes a chance de prejuízo do estado nutricional. Além disso,indivíduos com demência avançada apresentaram risco 3,7 vezes maior de subnutrição do que idosos em estágio inicial da doença, concordando com resultados de outros autores, em que se observa que a subnutrição está associada com a gravidade da demência. ${ }^{18}$

Uma revisão recente afirmou que a perda ponderal representa não somente um fator de risco para demência, como também tende a ser considerado um dos sinais clínicos e está associada com sua progressão. ${ }^{6}$ Algumas hipóteses foram elaboradas na tentativa de justificar essa alteração ponderal observada entre os idosos com demência.

A atrofia de regiões cerebrais relacionadas com a demência podem envolver áreas cerebrais responsáveis pelo controle do apetite e do balanço energético, incluindo o córtex temporal mesial (CTM), o hipotálamo e o giro cingulado. ${ }^{19}$

Em estágios iniciais da doença, em razão da disfunção da memória e do agravo da capacidade funcional, os idosos demenciados podem apresentar dificuldade em adquirir, armazenar e preparar os alimentos, podendo até esquecer que já se alimentaram. ${ }^{6}$

Com a progressão da demência, a apraxia de membros e a apraxia oral fazem com que o idoso tenha dificuldade em usar os talheres, interferindo também na manipulação do alimento em cavidade oral, o que resulta em atraso da 
iniciação da deglutição, com consequente dificuldade faríngea e risco de aspiração. ${ }^{20}$

Os problemas de mastigação e deglutição, associados à perda de dentição e disfagia, podem prejudicar ainda mais a ingestão alimentar desses pacientes, uma vez que essas queixas podem limitar o consumo de alimentos sólidos como frutas, vegetais e carnes. Por outro lado, a adequação da consistência das refeições, com oferta de dieta pastosa, por exemplo, pode diminuir o aporte diário de energia e nutrientes. ${ }^{4,21}$ Entretanto, no presente, esses fatores não foram associados à subnutrição na análise ajustada para idade, gênero e estágio da demência, o que pode ressaltar o efeito da gravidade da doença como determinante do estado nutricional.

Sintomas psicológicos, observados nos estágios inicial e moderado da doença, também podem interferir na ingestão alimentar; a apatia está associada com redução no interesse pela alimentação enquanto a tristeza e os sintomas depressivos podem resultar em hiporexia. ${ }^{22}$

A disfunção executiva o torna socialmente inapropriado quanto ao comportamento durante a refeição e à escolha do alimento, além de influenciar no tempo da alimentação.Já as alterações de linguagem dificultam a expressão da preferência do alimento e a compreensão das informações a respeito da refeição, ocasionando problemas na interpretação do comportamento alimentar do idoso. ${ }^{23}$

Nesse contexto, os cuidadores constituem peça fundamental para todo o tratamento do idoso com demência, em especial no que diz respeito ao cuidado nutricional. Os dados deste estudo revelam que os idosos demenciados eram cuidados pelos filhos, cônjuges ou cuidador formal, confirmando a dependência desses idosos para realizar suas atividades.

Alguns estudos sugerem que tanto a quantidade quanto a qualidade dos cuidados à disposição dos idosos no momento das refeições podem influenciar a ingestão de alimentos e seu peso corporal. ${ }^{24}$ De acordo com o estudo de Lin et al., ${ }^{25}$ os idosos com demência avançada apresentam melhor ingestão alimentar quando recebem maior auxilio durante a alimentação ou quando as refeições são feitas na presença de familiares.

No entanto, a sobrecarga de tarefas e cuidados atribuídos ao cuidador, somada às mudanças de comportamento alimentar do idoso demenciado, podem também torná-lo incapaz da atenção e paciência necessárias para dieta assistida ao idoso. ${ }^{26}$

No presente estudo, houve diferença significativa entre os grupos quanto à dependência para alimentação, embora esse resultado não tenha sido confirmado pela análise de regressão ajustada para idade, gênero e estágio da demência. Ainda assim, considera-se que a presença do cuidador durante a avaliação nutricional e a elaboração de estratégias nutricionais para domicílio são fundamentais para o sucesso da intervenção nutricional. ${ }^{27}$
O prejuízo do estado nutricional pode, ainda, ser ocasionado pelo aumento das necessidades nutricionais, em razão da elevação dos níveis de cortisol devido a um estado hipercatabólico, associado ao maior nível de atividade física durante a exacerbação de transtornos de comportamento, com agitação e agressividade ou pelos distúrbios motores. ${ }^{28}$

$\mathrm{O}$ uso de múltiplos medicamentos também poderia interferir diretamente no estado nutricional de idosos com demência, haja vista sua interferência direta no paladar, olfato e sintomas gastrintestinais. ${ }^{29}$ No entanto, no presente estudo não foi encontrada associação direta com a subnutrição.

Certas complicações, como a úlcera por pressão, mais evidente nos estágios avançados da demência, pode agravar o estado nutricional dos idosos, uma vez que aumentam as necessidades nutricionais. ${ }^{30}$ No entanto, pelo pequeno número de idosos que apresentavam úlcera por pressão, não foi possível avaliar associação entre sua presença e a subnutrição.

Portanto, apesar de não haver consenso entre os fatores que determinam a perda ponderal nos idosos com demência, é fato que ela agrava o prognóstico da doença e a qualidade de vida desses indivíduos. ${ }^{30}$

O presente estudo, embora apresente resultados que não podem ser considerados como referência, mas como característicos da população estudada, em função do tipo de estudo realizado - descritivo e transversal - abre espaço para novas pesquisas. Além disso, em razão do tamanho amostral reduzido, não foi possível caracterizar o estado nutricional de idosos com demências de diferentes etiologias, em cada fase da doença.

\section{CONCLUSÃO}

A subnutrição foi identificada em mais da metade dos idosos com demência estudados, sendo que o comprometimento cognitivo avançado e a perda ponderal recente estão diretamente associados com o prejuízo do estado nutricional desse grupo de idosos.

Dessa forma, este estudo ilustra a importância de se proceder a avaliação nutricional precoce e periódica do idoso com demência, para que efetivas intervenções dietéticas sejam implementadas, com o objetivo de manter ou recuperar o estado nutricional, melhorar a qualidade de vida, auxiliar na preservação das funções remanescentes e, assim, retardar o aparecimento da subnutrição e suas complicações.

\section{CONFLITO DE INTERESSES}

Os autores declaram não ter havido conflito de interesses. 


\section{REFERÊNCIAS}

1. Solfrizzi V, Panza F, Frisardi V, Seripa D, Logroscino G, Imbimbo BP, et al. Diet and Alzheimer's disease risk factors or prevention: the current evidence. Expert Rev Neurother. 2011;11(5):677-708.

2. Prince M, Bryce R, Albanese E, Wimo A, Ribeiro W, Ferri CP. The global prevalence of dementia: a systematic review and metaanalysis. Alzheimer's Dementia. 2013;9(1):63-75.

3. Guigoz Y. The Mini Nutritional Assessment (MNA) review of the literature: what does it tell us? J Nutr Health Aging. 2006;10(6):466-85.

4. Machado J, Caram CLB, Frank AA, Soares EA, Laks J. Estado nutricional na doença de Alzheimer. Rev Assoc Med Bras. 2009;55(2):188-91.

5. Zekry D, Herrmann FR, Grandjean R, Meynet MP, Michel JP, Gold G, et al. Demented versus non-demented very old inpatients: the same comorbidities but poorer functional and nutritional status. Age Ageing. 2008;37(1):83-9.

6. Sergi G, De Rui M, Coin A, Inelmen EM, Manzato E. Weight loss and Alzheimer's disease: temporal and aetiologic connections. Proc Nutr Soc. 2013;72(1):160-5.

7. Gillette-Guyonnet S, Nourhashémi F, Andrieu S, Glisezinski I, Ousset PJ, Rivière D, et al. Weight loss in Alzheimer disease. Am J Clin Nutr. 2000;71(2):6375-642S.

8. American Psichiatric Association. Diagnostic and statistical manual of mental disorders. 4th ed. Washington: APA; 1997.

9. Morris JC. The Clinical Dementia Rating (CDR): current version and scoring rules. Neurology. 1993;43(11):2412-4.

10. Duarte ACG. Avaliação nutricional: aspectos clínicos e laboratoriais. Rio de Janeiro: Atheneu; 2007.

11. Chumlea WC, Roche AF, Steinbaugh ML. Anthropometric approaches to the nutritional assessment of the elderly. In: Munro H, Danford DE. Human nutrition: nutrition, aging, and the elderly. New York: Plenum Press; 1989. p. 335-361.

12. Chumlea WC, Roche AF, Steinbaugh ML. Estimating stature from knee height for persons 60 to 90 years of age. J Am Geriatr Soc. 1985;33(2):116-20.

13. Barbosa AR, Souza JMP, Lebrão ML, Laurenti R, Marucci MFN. Anthropometry of elderly residents in the city of São Paulo, Brazil. Cad Saúde Pública. 2005;21(6):1929-38.

14. Omran ML, Morley JE. Assessment of protein energy malnutrition in older persons, Part II: Laboratory evaluation. Nutrition. 2000;16(2):131-40.

15. Christensson L, Unosson M, Ek AC. Evaluation of nutritional assessment techniques in elderly people newly admitted to municipal care. Eur J Clin Nutr. 2002;56(9):810-8.

16. Pan-American Health Organization. XXXVI Reunión del Comité Asesor de Investigaciones en Salud - Encuestra Multicentrica - Salud Bienestar y Envejecimeiento (SABE) en América Latina e el Caribe. Preliminary note; 2002.

17. Duchini L, Jordão AA, Brito TT, Diez-Garcia RW. Avaliação e monitoramento do estado nutricional de pacientes hospitalizados: uma proposta apoiada na opinião da comunidade científica. Rev Nutr. 2010;23(4):513-22.

18. Vellas B, Lauque S, Gillette-Guyonnet S, Andrieu S, Cortes F, Nourhashémi F, et al. Impact of nutritional status on the evolution of Alzheimer's disease and on response to acetylcholinesterase inhibitor treatment. J Nutr Health Aging. 2005;9(2):75-80.

19. Roberts SB, Rosenberg I. Nutrition and aging: changes in the regulation of energy metabolism with aging. Physiol Rev. 2006;86(2):651-67.

20. Abreu ID, Forlenza OV, Barros HL. Demência de Alzheimer: correlação entre memória e autonomia. Rev Psiquiatr Clín. 2005;32(3):131-6.

21. Viganó PC, Silva NS, Cremonezi CJ, Vannucchi GP, Campanelli MM. Variation in the energy and macronutrient contents of texture modified hospital diets. Rev Chil Nutr. 2011;38(4):451-7.

22. Prince M, Albanese E, Guerchet M, Prina M. Nutrition and dementia: a review of available research. London: Alzheimer's Disease International; 2014.

23. Athlin RNE, Norberg A, Asplund K. Caregiver's perceptions and interpretations of severely demented patients during feeding in a task assignment system. Scand J Caring Sci. 1990;4(4):147-55.

24. Lecheta DR, Schieferdecker MEM, Mello AP, Berkenbrock I, Neto JC, Maluf EMCP. Dietary inadequacies in the elderly with Alzheimer's disease followed at the reference health center for elderly care in Curitiba, Brazil. J Aging Res Clin Practice. 2015;4(2):85-91.

25. Lin LC, Watson R, Wu SC. What is associated with low food intake in older people with dementia? J Clin Nurs. 2008;19(1-2):53-9.

26. Rivière S, Gillette-Guyonnet S, Andrieu S, Nourhashemi F, Lauque S, Cantet $C$, et al. Cognitive function and caregiver burden: predictive factors for eating behaviour disorders in Alzheimer's disease. Int $J$ Geriatr Psychiatry. 2002;17(10):950-5.

27. Fagerström C, Palmqvist R, Carlsson J, Hellström Y. Malnutrition and cognitive impairment among people 60 years of age and above living in regular housing and in special housing in Sweden: a populationbased cohort study. Int J Nurs Stud. 2011;48(7):863-71.

28. Smith KL, Greenwood CE. Weight loss and nutritional considerations in Alzheimer disease. J Nutr Elder. 2008;27(3-4):381-403.

29. Kishi T, Elmquist JK. Body weight is regulated by the brain: a link between feeding and emotion. Mol Psychiatry. 2005;10(2):132-46.

30. Martín MAC, Ortega SB, Rodríguez LD, Muiño CC, Silleras BM, Del Río MPR. Presencia de malnutrición y riesgo de malnutrición en ancianos institucionalizados con demencia en función del tipo y estadío evolutivo. Nutr Hosp. 2012;27(2):434-40. 